\title{
Place de la sociologie dans l'ordre des sciences
}

Claude Grignon

\section{OpenEdition}

\section{Journals}

Édition électronique

URL : http://journals.openedition.org/ress/137

DOI : $10.4000 /$ ress. 137

ISSN : 1663-4446

\section{Éditeur}

Librairie Droz

\section{Édition imprimée}

Date de publication : 1 septembre 2008

Pagination : 91-105

ISBN : 978-2-600-01242-3

ISSN : 0048-8046

Référence électronique

Claude Grignon, «Place de la sociologie dans l'ordre des sciences », Revue européenne des sciences sociales [En ligne], XLVI-142 | 2008, mis en ligne le 01 septembre 2011, consulté le 19 avril 2019. URL http://journals.openedition.org/ress/137 ; DOI : 10.4000/ress.137 


\section{CONCLUSION}

\section{PLACE DE LA SOCIOLOGIE DANS L'ORDRE DES SCIENCES}

Je me suis efforcé de dissiper une confusion qui dispense les sciences de l'homme de soumettre leurs théories à l'épreuve de la réalité, et qui compromet leur avenir en les empêchant de se mettre à la recherche des voies par lesquelles elles pourraient s'acquitter de cette obligation commune à toutes les sciences; c'est le cas en particulier de la sociologie, dont la survie n'est sans doute pas plus assurée que son apparition n'était probable.

Cette confusion s'introduit à la faveur des possibilités d'inversion et de retournement que présentent les précautions et les dispositions spéciales réclamées par la nature particulière des difficultés que rencontrent les sciences de l'homme, notamment dans les rapports entre l'interprétation et l'observation: dans la modélisation qui précède et qui accompagne tout constat et toute description; dans la définition initiale des objets et du point de vue sous lequel on les envisage; dans la détermination des caractères-clés qui orientent et guident l'observation et décident, aux niveaux successifs de la généralisation, de la constitution des catégories et des types. Elle naît et profite aussi de l'obligation de recourir à l'association des idées, des mots et des images, et au pouvoir d'évocation du langage naturel pour la formation des concepts et pour la reconstitution narrative des processus. Ces risques sont communs à toutes les sciences de l'observation et à toutes les sciences historiques; les sciences expérimentales elles-mêmes n'en sont pas complètement exemptes. Mais les sciences de l'homme collectif, surtout les plus générales, comme la sociologie, y sont plus exposées que les sciences de la nature, parce que l'ordre de réalité qu'elles étudient se définit par un niveau supérieur d'organisation, et parce qu'il n'oppose pas de résistance matérielle à leurs interprétations et à leurs représentations. Cette immunité interprétative les renvoie du côté des savoirs herméneutiques. Les pseudo-théories s'y développent en redoublant de rouerie sophistique sous couvert de déjouer les ruses de la complexité, apparente ou réelle, avec laquelle elles prétendent rivaliser; on croit ainsi «rendre compte» de la confusion du réel en la reproduisant, au lieu de tout faire pour la dissiper en étant d'autant plus minutieux dans l'observation, plus rigoureux dans le raisonnement, plus clair dans l'expression que l'objet étudié paraît plus embrouillé et plus retors.

En obligeant les sciences historiques et observationnelles à s'aligner sur l'épistémologie idéalisée des sciences nomothétiques, le réductionnisme leur impose des obligations auxquelles les propriétés des phénomènes qu'elles cherchent à expliquer leur interdisent de satisfaire et leur assigne des buts que ces propriétés les empêchent d'atteindre. Cette aliénation les enferme dans le statut de 
sciences par défaut: on ne voit dans les pratiques de recherche et dans les façons de théoriser qui leur sont propres que des particularismes, des dérogations et des manquements à la norme. Elle les empêche de prendre pleinement et clairement conscience des questions que les obstacles et les difficultés propres à l'ordre de réalité dont elles s'occupent invitent à poser ou à reformuler. Les sciences de l'homme y sont les plus exposées; en leur imposant une norme épistémologique irréaliste et inappropriée, on les décourage, on les dissuade de chercher à être, dans leur ordre, pleinement des sciences. Comme elles ne peuvent renoncer complètement à être des sciences, sous peine de perdre leur position académique et leur autorité dans les débats politico-intellectuels, il ne reste plus qu'à imaginer et à aménager une niche où l'on passerait en continu de la non science aux quasi sciences et des quasi sciences à la science (où s'établissent entre autres les spécialistes de la «sociologie lucrative », qui vivent et prospèrent aux dépens et au détriment de la sociologie). C'est ainsi que W. Lepenies «dépasse» l'opposition entre la culture scientifique et la culture des intellectuels littéraires signalée et déplorée par C. P. Snow, en reprenant l'idée d'une «troisième culture» avancée par ce dernier d'une manière assez vague, dans une perspective volontariste et militante plutôt qu'épistémologique (Chazel 1993) ${ }^{1}$. Dans la hiérarchie réductionniste les sciences de l'homme collectif sont les plus éloignées de l'idéal représenté par les mathématiques et la physique; mais elles n'en sont séparées que par des différences de degré (dans le scénario optimiste de Goblot, elles peuvent espérer les rejoindre). On ménage ainsi la possibilité d'une double appartenance, d'une sociologie, d'une histoire ou d'une ethnologie qui, tout en étant très philosophiques ou très littéraires seraient encore, malgré tout, si peu que ce soit, scientifiques. Les sciences de l'homme deviennent alors des «sciences humaines »"; on passe sans s'en apercevoir de la non-science à la science, ou plutôt il n'y a pas lieu de s'en apercevoir puisqu'il n'y a plus de solution de continuité, plus de démarcation entre la science et les autres produits de l'activité intellectuelle.

Dans ces conditions, on ne cesse d'être réductionniste que pour devenir relativiste. Rien de plus opposé, pourtant, que les humeurs et que les courants d'idées

1 W. Lepenies, Les trois cultures, entre science et littérature, l' avènement de la sociologie, Paris, Editions de la Maison des Sciences de l'Homme, 1990. La «troisième culture » de Snow est un «courant d'opinion», qui «groupe des intellectuels exerçant leur activité dans des disciplines très variées: histoire sociale, sociologie, démographie, sciences politiques, sciences économiques, psychologie, médecine, etc., ainsi qu'arts 'sociaux' comme l'architecture (...) Il est sans doute encore trop tôt pour affirmer qu'une troisième culture nous est déjà née. Mais je suis à présent convaincu que le jour approche où elle naîtra. Certaines difficultés de communication se trouveront, de ce fait, aplanies: car cette culture devra nécessairement, ne fût-ce que pour remplir son office, entretenir des relations avec la culture scientifique». C. P. Snow, Supplément aux deux cultures, in Les deux cultures, op. cit. , pp. 107, 108. Pour une critique approfondie du livre de W. Lepenies, cf. F. Chazel, «L'esthétisme sceptique et ses limites en histoire de la sociologie », Revue française de sociologie, XXXIV, 1993, pp. 347-349.

2 Toutes les sciences sont (trop) humaines. Même si elles sont réputées moins dures, et donc plus douces, les sciences de l'homme ne sont pas plus humaines que les sciences de la nature ne sont naturelles. On confond une science, ou une discipline avec son objet quand on dit, à tort, sciences sociales (comme en anglais), sciences politiques (mais en allemand Politologie), sociologie rurale (et pourquoi pas rustique), sociologie urbaine, sociologie religieuse (et pourquoi pas dévote); mais on dit, mieux, sociologie du travail (et non laborieuse), sociologie, ou histoire de l'éducation (et non scolaires), sociologie de l'alimentation (et non alimentaire), sociologie ou histoire de l'art, etc. 
auxquels se rattachent respectivement le réductionnisme et le relativisme: foi dans la science, optimisme rationaliste poussé jusqu'au logicisme pour le premier, réaction contre le rationalisme et contre la science, nihilisme cognitif dans le cas du second ${ }^{3}$. La conception philosophique, en fait religieuse, de la vérité, comme savoir absolu et vérité révélée, que vise le relativisme est en opposition complète avec la conception scientifique de la vérité, conquise et découverte, progressive et toujours provisoire, c'est-à-dire proprement relative. Cette fiction ad hoc est au principe du relativisme: dès lors que la vérité est une vérité parfaite, que les sciences ne peuvent atteindre, il n'y a plus de sciences, ou du moins la science ne se distingue plus de la non-science. Mais on peut confondre cet absolutisme avec l'intransigeance scientifique qui veut distinguer nettement, sans compromis, la vérité de l'erreur. Par ailleurs, chercher à expliquer ce qui va de soi, c'est le relativiser; sous ce rapport le réductionnisme est l'expression radicale d'un relativisme scientifique qui est lui-même l'héritier et le prolongement du relativisme philosophique. La curiosité scientifique ne met pas seulement en question ce qui est communément admis, doxas ou dogmes, elle s'interroge sur les choses et les événements dont l'existence de fait s'impose à un point tel qu'on ne les remarque pas (par exemple sur la pesanteur à laquelle, comme le notait A. Comte, nulle culture n'a songé à attribuer un dieu). Le refus du surnaturel, la volonté de naturaliser tous les phénomènes et pour cela de les mettre tous au même niveau conduisent à rejeter la notion d'ordres et de hiérarchie entre des ordres; par crainte de revenir à la conception métaphysique ou religieuse des ordres (ordre divin, ordre humain, ordre de la grâce, etc.), on se refuse à diviser la nature, ou la réalité, en ordres séparés (ordre de la matière, ordre du vivant, et en effet, là encore, ordre humain). La révolution darwinienne désacralise l'homme en postulant et en reconstituant la continuité biologique et historique entre l'homme et l'animal. Comme le remarquent Durkheim et Mauss, l'esprit critique qui anime l'esprit scientifique aboutit ainsi, jusque dans les sciences de l'observation, à un continuisme qui prête à confusion avec le continuisme primitif, caractéristique de la pensée magique:

Pour nous (...) classer les choses, c'est les ranger en groupes distincts les uns des autres,
séparés par des lignes de démarcation nettement déterminées. De ce que l'évolution-
nisme moderne nie qu'il y ait entre eux un abîme infranchissable, il ne s'ensuit pas qu'il
les confonde jusqu'à réclamer le droit de les déduire les uns des autres. Il y a, au fond de
notre conception de la classe, l'idée d'une circonscription aux contours arrêtés et définis.
(...) Non seulement notre notion actuelle de la classification a une histoire, mais cette
histoire elle-même suppose une préhistoire considérable. On ne saurait, en effet, exagé-
rer l'état d'indistinction d'où l'esprit humain est parti. Même aujourd'hui, toute une
partie de notre littérature populaire, de nos mythes, de nos religions, est basée sur une
confusion fondamentale de toutes les images, de toutes les idées. Il n'en est pas pour ainsi
dire qui soient, avec quelque netteté, séparées des autres. Les métamorphoses, les trans-
missions de qualités, les substitutions de personnes, d'âmes et de corps, les croyances
relatives à la matérialisation des esprits, à la spiritualisation d'objets matériels, sont des
éléments de la pensée religieuse ou du folklore. Or l'idée même de semblables transmu-

3 Sur les origines intellectuelles du relativisme (Thoreau, Shelley, Coleridge, Wordsworth, Blake, Goethe, Rousseau, Vico, Montaigne) et du logicisme, cf. G. Holton, The Scientific Imagination, op. cit., pp. 87 sq; sur la réaction romantique contre le rationalisme des Lumières, cf. H. Brunschwig, Société et romantisme en Prusse au XVIII e siècle, Paris, Flammarion, 1973. 
tations ne pourrait pas naître si les choses étaient représentées dans des concepts délimités et classés. Le dogme chrétien de la transsubstantiation est une conséquence de cet état d'esprit et peut servir à en prouver la généralité (Durkheim et Mauss 1903) ${ }^{4}$.

Le réductionnisme a donc en commun avec le relativisme de reposer sur une conception continuiste (et nominaliste) de la connaissance; du moins est-ce le cas du «réductionnisme explicatif», qui postule à la fois la continuité ontologique et la continuité cognitive du réel, et du «réductionnisme théorique», qui vise à unifier la science en ramenant la diversité des phénomènes aux lois élémentaires qui régissent la matière ${ }^{5}$. Dans cette perspective, on s'efforce d' "expliquer complètement la vie sans la vie », de réduire la biologie à la physique et à la chimie (la chimie étant elle-même de plus en plus ramenée à la physique); la division de la réalité en ordres séparés n'est qu'une commodité provisoire, qui repose sur une illusion destinée à disparaître à mesure que la science progresse et parvient de plus en plus à «expliquer du visible compliqué par de l'invisible simple » (Canguilhem 1985: 68, 94) 6 . Comme le dit A. Comte (que cette remarque dispense de se prononcer sur la question, «métaphysique», de l'identité ou de la différence de nature entre les ordres), «les corps organisés », qui sont «plus compliqués et plus particuliers que les autres (...) dépendent des précédents, qui, au contraire, n'en dépendent nullement $»^{7}$. L'ordre de base pourrait exister indépendamment des

4 E. Durkheim, M. Mauss, «De quelques formes de classification - contribution à l'étude des représentations collectives.» Année sociologique, 6, (1903).

5 Sur les différentes espèces de réductionnisme, cf. E. Mayr, Histoire de la biologie, op. cit., pp. 69 sq. Le réductionnisme explicatif «affirme que l'on ne peut comprendre un tout avant qu'on l'ait décomposé en ses différentes parties, et celles-ci, à leur tour, en leur composantes, et ainsi de suite jusqu'au plus petit niveau hiérarchique d'intégration »; le réductionnisme théorique entend «montrer que des théories et des lois, formulées dans un domaine scientifique donné (généralement un domaine plus complexe ou plus élevé dans la hiérarchie), représentent des cas particuliers de théories et de lois formulées dans un autre domaine de la science. Lorsqu'on arrive à faire cette démonstration, on dit qu'une branche de la science a été 'réduite' à une autre (...) Pour prendre un exemple spécifique, on considère que la biologie serait réduite à la physique, si l'on arrivait à définir tous les termes de la biologie en termes de physique, et si toutes les lois de la biologie pouvaient être déduites des lois de la physique». (C'est le scénario de Goblot).

6 C'était le programme de Jean Perrin («expliquer du visible compliqué...»), ou de Jean Rostand: «Le mécanisme a, à l'heure actuelle, une position extrêmement solide, et l'on ne voit guère ce qu'on peut lui répondre quand, fort de ses succès quotidiens, il demande simplement des délais pour achever son œuvre, à savoir pour expliquer complètement la vie sans la vie » (cités par G. Canguilhem, La connaissance de la vie, Paris, Vrin, 1985, pp. 68, 94). La biophilosophie, et la biologie même, continuent d'être partagées entre le mécanisme matérialiste d'un Haeckel, qui pense «qu'on trouvera dans la chimie du carbone et des dérivés du carbone (...) l'explication scientifique de la formation sur terre de l'organisme ancestral qui fut à l'origine des lignées vivantes », et le vitalisme finaliste d'un Driesch, pour qui «l'organisation vitale» s'explique par une «élaboration structurale relativement indépendante des éléments matériels». A. Fagot-Largeault, in D. Andler, A. Fagot-Largeault, B. Saint-Sernin, Philosophie des sciences, op. cit., pp. 528-535. Cf. aussi F. Jacob, sur l'opposition entre l'attitude «intégriste» qui «refuse de considérer que toutes les propriétés d'un être vivant, son comportement, ses performances peuvent s'expliquer par ses seules structures moléculaires » et l'attitude réductionniste pour qui «l'organisme est bien un tout, mais qu'il faut expliquer par les seules propriétés des parties ». La logique du vivant, une histoire de l' hérédité, Paris, Gallimard, 1970, pp. 14, 15.

7 A. Comte, Cours de philosophie positive, $2^{\mathrm{e}}$ leçon; cité par A. Fagot-Largeault, in D. Andler, A. Fagot-Largeault, B. Saint-Sernin, Philosophie des sciences, op. cit., p. 502. 
deux autres; inversement, l'ordre du vivant suppose l'existence de l'ordre de la matière inerte; l'ordre humain repose sur l'ordre du vivant, et, par l'intermédiaire de celui-ci, sur l'ordre de la matière inerte. C. Bernard s'en tient pour sa part à l'idée que la condition nécessaire des ordres supérieurs est dans les ordres inférieurs, ce qui sauvegarde l'autonomie des premiers (Bernard 1925 [1867]) ${ }^{8}$. Mais, pour peu que l'on confonde condition nécessaire et condition suffisante et que l'on glisse ainsi de l'idée de condition à celle de cause, la hiérarchie des ordres s'inverse: on en arrive à l'idée que l'inférieur «explique» le supérieur. Le réductionnisme n'a sans doute pas l'exclusivité de «l'exigence d'explication analytique », mais celle-ci le caractérise en propre. Elle conduit à entrer toujours plus avant dans le détail et donc dans la différenciation, à faire des distinctions toujours plus fines, à introduire sans cesse des discontinuités nouvelles; mais du même coup, elle réduit de plus en plus les intervalles, elle suppose qu'entre deux états contigus, il existe quantité, en fait une infinité d'états intermédiaires. Elle aboutit ainsi, paradoxalement, à réduire, à effacer les discontinuités.

La conception réductionniste de l'unité de la science ne garantit donc nullement la spécificité de la connaissance scientifique dans «l'empire universel des représentations» (Imbert 1998) ${ }^{9}$. Pour que les différentes sciences puissent répondre, chacune à leur manière et par les moyens qui leur sont propres, aux exigences communes à toutes les sciences et par lesquelles la science, dans son ensemble, se distingue des autres représentations du monde - rechercher les démentis de la réalité pour trouver les causes des phénomènes -, il faut au contraire qu'elles conçoivent et développent des épistémologies particulières, adaptées à la diversité de leurs besoins et des contraintes auxquelles elles sont soumises $^{10}$. On retrouve ici la question des épistémologies régionales. A vrai dire,

${ }^{8}$ «Si dans une horloge électrique, par exemple, on enlevait l'acide de la pile, on ne concevrait pas que le mécanisme continuât de marcher; mais, si l'on restituait ensuite convenablement l'acide supprimé, on ne comprendrait pas non plus que le mécanisme se refusât à reprendre son mouvement. Cependant, on ne se croirait pas obligé pour cela de conclure que la cause de la division du temps en heures, en minutes, en secondes, indiquées par l'horloge, réside dans les qualités de l'acide ou dans les propriétés du cuivre ou de la matière qui constitue les aiguilles et les rouages du mécanisme. (...) En résumé, nous n'avons à constater dans tout ce qui précède que les conditions d'un déterminisme physico-chimique nécessaire pour la manifestation des phénomènes vitaux aussi bien que pour la manifestation des phénomènes minéraux. Nous ne saurions donc y chercher des explications qui aboutiraient à un matérialisme absurde ou vide de sens ». «Quant aux phénomènes de la vie, j'admets que ces phénomènes, considérés dans leurs formes diverses de manifestation et dans leur nature intime, ont à la fois une spécialité de forme qui les distingue (...) et une communauté de lois qui les confond avec tous les autres phénomènes du monde cosmique». C. Bernard, «Le problème de la physiologie générale» (1867) in La science expérimentale, op. cit., p. 126-127, 113.

${ }^{9}$ C. Imbert, «Frege, entre Kant et Wittgenstein », in M. Marion et A. Voizard, Frege, Logique et philosophie, Paris, L'Harmattan, 1998, p. 287.

${ }^{10}$ Sur le physicalisme et le logicisme inhérents à l'idée de l'unité de la science, cf. A. FagotLargeault, Philosophie des sciences, Paris, Eyrolles, 2006: «Dans la première moitié du vingtième siècle, le paradigme dominant devient celui de l'unité de la science, promu par les positivistes du Cercle de Vienne (...). L'idée directrice est formulée par Carnap dans un texte célèbre, traduit en anglais sous le titre 'l'unité de la science'(«Die physicalische Sprache als Universalsprache der Wissenschaft», Erkenntnis, 1932, II, 4: 232-241; The Unity of Science, London, Kegan Paul, 1934). Le modèle des sciences est la physique mathématique. (...) L'unité (ou le projet d'unification) de la science, qui s'épanouit dans la série de l'Encyclopédie internationale des sciences unifiées, a un 
l'expression «épistémologies régionales » est mal choisie. Elle invite à penser la diversité épistémologique à partir de la représentation commune des divisions de l'espace, ou plus concrètement d'un territoire. Elle implique un fédéralisme épistémologique conçu à l'image du fédéralisme politique. «Revendiquer » une autonomie épistémologique, c'est faire sécession, c'est sortir de la Science comme on sort de la République, une et indivisible, ou de la Nation. Les tenants de l'unité peuvent sans doute concéder aux «partisans» des épistémologies régionales un certain degré d'autonomie; mais le «pouvoir central » demeure, détenu et exercé par les sciences les plus «dures», celles qui occupent la position dominante dans la hiérarchie communément admise des sciences. Dans cette perspective, toute typologie des sciences est une topologie, lourde de connotations géopolitiques; de la notion de démarcation, on revient à l'idée de frontière; la non-science est définie implicitement comme ce qui est extérieur, «étranger» à la science.

Plutôt que d'épistémologies régionales, on parlera donc d'épistémologies spécifiques, propres aux différentes sciences parce qu'appropriées à la relation que cellesci entretiennent avec leurs objets respectifs. D'un côté la science aspire à l'unité, ou plutôt à l'unification; mais par ailleurs, en pratique, elle tend vers une diversification sans cesse croissante; la spécialisation est à la fois la condition et la conséquence de son développement. Les sciences constituées sont des épistémologies spécifiques en acte; la diversification épistémologique existe de fait, dans l'organisation même de la science. Ce qui fait question, de ce point de vue, c'est que les épistémologies spécifiques fassent question, une question de philosophie des sciences et non une question scientifique. Il faut pourtant continuer à la poser, car si la science l'a résolue, c'est en pratique, en progressant, mais aussi en l'ignorant. Il faut pour commencer répondre à l'objection qui refuse les épistémologies spécifiques par crainte de s'engager dans une voie qui conduirait à la fragmentation et au morcellement indéfinis de la science, et pour finir à sa désintégration: si vous accordez le droit à la différence épistémologique, jusqu'où irez-vous dans la différenciation, à quel niveau de particularisme, de singularité descendrez-vous? On rappellera que les objets respectifs des différentes sciences obligent à certaines opérations et en interdisent d'autres, ou au moins les rendent plus difficiles; les procédés et les instruments d'investigation varient selon l'échelle des objets, en fonction des obstacles, et d'abord de la distance, qui les séparent de l'observateur. Possible sur les objets du monde physique qui sont à notre portée, sinon à notre échelle, l'expérimentation directe sur l'homme, et plus généralement sur les êtres vivants, est empêchée en sociologie, limitée en biologie, par des interdits moraux qui font partie de la réalité sociale; parmi les sciences de l'homme, il faut distinguer entre celles qui, comme la sociologie, peuvent créer leurs données par l'enquête, et celles qui, comme l'histoire, doivent les reconstituer à partir des fragments du passé qui leur sont parvenus, etc.

Mais, nous dira-t-on, c'est là se placer au niveau des techniques, au mieux à celui des méthodes, et non au niveau proprement épistémologique, qui serait celui des principes. Cette objection procède d'une conception de l'épistémologie déta-

double aspect: toutes les données scientifiques doivent pouvoir, en dernière analyse, s'exprimer dans le langage de la physique (c'est le physicalisme); et la théorie scientifique doit satisfaire à des critères logiques elle doit être, autant que possible, axiomatisée; elle ne doit pas être contradictoire, ni redondante, etc.». 
chée de la pratique des sciences. Le séminaire qui m'a amené à développer ces réflexions était un séminaire de recherche, c'est-à-dire un séminaire de chercheurs; nous avions en tête les questions classiques d'épistémologie théorique ou de philosophie des sciences, mais nous voulions les aborder et les traiter dans une perspective nouvelle, en partant de l'analyse des difficultés, des embarras et des obstacles, plus ou moins perçus, plus ou moins conscients, que rencontre la pratique de la recherche dans les différentes disciplines. Nous partions de l'idée qu'on ne peut savoir en quoi consiste et ce que vaut l'opus operatum que si l'on connaît le modus operandi, que pour parvenir à une connaissance scientifique de la science, à une véritable épistémè, il faut appliquer le raisonnement scientifique aux connaissances scientifiques, rechercher les conditions, les causes et les processus dont elles résultent. L'exemple du langage, à la fois instrument et mode de pensée, montre assez que les outils dont nous faisons usage ne sont jamais neutres. Il faut donc reprendre le programme bachelardien, appliquer la vigilance épistémologique à nos pratiques de recherche, à nos méthodes, à nos techniques pour savoir à quoi elles nous engagent, ce qu'elles nous conduisent à conjecturer et à concevoir, et, aussi bien, ce qu'elles nous empêchent de penser et de savoir; également pour débusquer les croyances inconscientes, les options métaphysiques qui pèsent sur nos préférences et sur nos choix de méthodes ${ }^{11}$.

On est fondé à distinguer des épistémologies spécifiques si et seulement si l'on peut distinguer des articulations, des divisions objectives de la réalité. La division de la nature en trois ordres, ordre physico-chimique, ordre du vivant, ordre humain, correspond aux grandes divisions de fait de la science; mais elle correspond aussi à une représentation et à une mise en ordre anthropocentriques du monde, à partir de l'homme qui s'octroie sa propre classe; viennent ensuite (moralement) les autres êtres vivants, animaux et végétaux, enfin (matériellement et, chronologiquement, au commencement) les choses. Il faut donc trouver des différences objectives entre ces trois ordres. Comme fondement de l'autonomie de l'ordre du vivant par rapport à l'ordre de la matière, et de l'autonomie de l'ordre anthropologique par rapport à l'ordre du vivant, on invoque souvent un degré, ou plutôt un niveau supérieur de complexité. A partir de là, deux possibilités :

1. Ces objets, «plus difficiles à connaître» parce que plus difficiles à simplifier, sont simplifiables; leur complexité se réduit à mesure que les sciences qui les

\footnotetext{
${ }^{11}$ La métaphore du «métier de sociologue» (plus généralement du métier de savant) a l'avantage de rappeler que la recherche est laborieuse, que la réflexion théorique est indissociable d'un ensemble de tâches et d'opérations dites empiriques. Assimiler la recherche à un artisanat intellectuel permet aussi d'insister sur l'indépendance du chercheur et des équipes de recherche par rapport aux institutions qui les emploient; dans le même ordre d'idées, on préférait naguère parler «d'atelier» plutôt que de séminaire. Mais on risque là encore de réactiver les oppositions qu'on prétend dépasser — entre l'artisan et l'artiste (ou l'intellectuel), entre le labeur et l'inspiration, entre le métier et la vocation, etc. C'est ainsi que Husserl déplore que les savants, «à cause de la spécialisation croissante de leur travail dans les sciences positives, [deviennent] de moins philosophes et de plus en plus des sortes d'artisans » (E. Husserl, La crise des sciences européennes et la phénoménologie transcendantale, op. cit., p. 16). De fait, une méthode éprouvée peut toujours dégénérer en routine; la spécialisation peut conduire à un usage automatique et aveugle des techniques. C'est pourquoi il faut s'interroger sur le sens de nos pratiques de recherche; en faisant de l'épistémologie appliquée, nous ne renonçons nullement à philosopher, nous philosophons, au sens sérieux du terme, mais nous philosophons en savants.
} 
étudient progressent, franchissent à leurs tours respectifs les étapes du chemin parcouru avant elles par les sciences de la matière. C'est, entre autres, on l'a vu, la position de Goblot. On revient au scénario réductionniste, dans lequel la complexité n'est qu'une illusion, qui résulte de l'avancement inégal des différentes sciences et qui se dissipe à mesure que les sciences de la vie et les sciences de l'homme rattrapent leur retard ${ }^{12}$.

2. La nature particulière de ces objets interdit de les simplifier au même degré et de la même manière que les objets étudiés par les sciences physiques. Pour les disciplines les moins avancées, qui sont aussi celles dont les objets sont, ou paraissent, les plus complexes, comme la sociologie, le choix entre ces deux possibilités est crucial. Malheureusement, en l'absence de critères communs à toutes les sciences, il est impossible de comparer les degrés de complexité de leurs objets respectifs. C'est en fonction d'une représentation de la réalité extérieure à la science qu'on peut supposer que les phénomènes physiques sont plus simples, et donc plus faciles à expliquer, que les faits biologiques ou que les faits historiques, psychologiques ou sociaux. Le degré de simplification auquel la physique est parvenue donne au contraire à penser que la simplicité des phénomènes physiques n'est qu'une apparence, et qu'ils sont en réalité d'une complication que l'expérience et la perception communes sont incapables d'entrevoir. Plus une science simplifie ses objets, et plus elle en fait ressortir la complexité cachée.

Pour E. Mayr, «la complexité ne différencie pas les systèmes organiques et inorganiques». Même si «les systèmes du monde vivant sont en moyenne infiniment plus complexes que ceux du monde inanimé », «il y a des systèmes inanimés hautement complexes (par exemple, les masses d'air en mouvement du système climatique mondial, ou bien les galaxies), et il existe un tout petit nombre de systèmes organiques relativement simples, comme certaines macromolécules ${ }^{13}$. Mais les systèmes vivants ne sont pas seulement complexes, ils sont organisés; ou plutôt la complexité, dans leur cas, va de pair, se confond avec l'organisation. «La plupart des structures de l'organisme sont dépourvues de sens hors de l'organisme: les ailes, les jambes, les têtes, les reins, ne peuvent vivre par eux-mêmes, mais seulement en tant que parties de l'ensemble» (Mayr 1989: 63, 64) ${ }^{14}$. L'impossibilité de négliger le niveau de l'organisme distingue la science des «corps vivants » de celle des «matières brutes » (Bernard 1925 [1867]: 118) ${ }^{15}$. Elle oblige

${ }^{12}$ Cf. C. Grignon, Complexity and Reductionism in Sociology, communication au colloque «The Science of Complexity: Chimera or Reality?», Arcidosso, 2-5 septembre 2003.

${ }^{13}$ En outre «on peut continuer à considérer certains systèmes relativement simples, tel le système solaire comme complexes même après que l'on ait réussi à en expliquer la complexité». E. Mayr, Histoire de la biologie, op. cit., pp. 63-64.

14 E. Mayr, id., p. 64.

15 «Le physiologiste a affaire à des procédés particuliers qui sont inhérents à la matière organisée, et qui constituent par conséquent l'objet spécial de ses études. La physiologie générale se trouve ainsi ramenée à être la science expérimentale qui étudie les propriétés de la matière organisée et explique les procédés et les mécanismes des phénomènes vitaux, comme la physique et la chimie expliquent les procédés et les mécanismes des phénomènes minéraux.» $\mathrm{C}$. Bernard, «Le problème de la physiologie générale», art. cit., p. 118. 
à opter pour une approche holistique, à admettre que les organismes sont des totalités qui ne se laissent pas réduire à la somme, ni même à l'agencement de leurs éléments. Par ailleurs, chaque organe se définit par sa fonction dans l'organisme: «chaque partie a une signification adaptative, et peut desservir des activités téléonomiques. Ces adaptations mutuelles des parties sont inconnues dans le monde inanimé $»^{16}$. Pour expliquer les phénomènes biologiques, il faut donc faire appel, plus ou moins ouvertement, à la cause finale, au risque de revenir au vitalisme et au finalisme ${ }^{17}$. Pour éviter que le rejet du réductionnisme explicatif ne ramène en deçà de la démarche analytique, il faut le distinguer du « réductionnisme constitutif », qui «affirme que la composition matérielle des organismes ne diffère en rien de ce que l'on trouve dans le monde inorganique » (Mayr 1989: 69) ${ }^{18}$.

Des cellules aux tissus, des tissus aux organes, des organes à l'organisme, la hiérarchie des niveaux d'organisation est une des caractéristiques essentielles des êtres vivants. Chacun de ces niveaux détermine un ordre d'explication qui lui est propre: «A chaque niveau, il y a différents problèmes, différentes questions auxquelles répondre, et différentes théories à formuler». Si la biologie est partagée entre des disciplines dont les références épistémologiques se distribuent entre les pôles opposés du holisme et du réductionnisme, c'est que ses divisions correspondent à «la structure hiérarchique des systèmes vivants». Chaque niveau d'organisation «a donné naissance à des branches distinctes de la biologie: les molécules à la biologie moléculaire, les cellules à la cytologie, les tissus à l'histologie, etc., jusqu'à la biogéographie et l'étude des écosystèmes (...) Ce sont les intérêts propres d'un chercheur qui le pousseront à se tourner vers une branche ou une autre. Un biologiste moléculaire n'est tout simplement pas intéressé par les problèmes qu'étudie le morphologiste ou le zoogéographe, et vice-versa. Les problèmes et les résultats des autres disciplines ne sont généralement pas pertinents pour les chercheurs qui travaillent à un niveau hiérarchique donné. Pour comprendre les phénomènes du vivant, il est nécessaire d'étudier tous les stades mais (...) les résultats trouvés aux strates inférieures contribuent peu à la solution des problèmes posés à de plus hauts niveaux» (Mayr 1989: 74) ${ }^{19}$. A chaque niveau supérieur d'organisation, on voit apparaître, «émerger» des propriétés

${ }^{16}$ E. Mayr, Histoire de la biologie, op. cit. p. 64.

${ }^{17}$ On trouve des traces «d'étonnement téléologique» (Schopenhauer) jusque chez C. Bernard: «Quand il s'agit d'une évolution organique qui est dans le futur, nous ne comprenons plus cette propriété de matière à longue portée. L'œuf est un devenir, il représente une sorte de formule organique qui résume l'être dont il procède et dont il a gardé le souvenir évolutif (...) Quand on observe l'évolution ou la création d'un être vivant dans l'œuf, on voit clairement que son organisation est la conséquence d'une loi organogénétique qui préexiste d'après une idée préconçue, et qui s'est transmise par tradition organique d'un être à l'autre. On pourrait trouver dans l'étude expérimentale des phénomènes d'histogenèse et d'organisation la justification des paroles de Goethe, qui compare la nature à un grand artiste. C'est qu'en effet la nature et l'artiste semblent procéder de même dans la manifestation de l'idée créatrice de leur œuvre». C. Bernard, «Le problème de la physiologie générale», art. cit., pp. $133,134$.

${ }^{18}$ D'après E. Mayr, les biologistes actuels adhèrent pratiquement tous au réductionnisme constitutif, et donc «ne sont pas des vitalistes, quoiqu'en disent certains philosophes », même s'ils rejettent les autres formes de réductionnisme». E. Mayr, Histoire de la biologie, op. cit., pp. 69, 70.

19 Id., p. 74. 
nouvelles et imprévisibles; la reconnaissance de l'émergence implique le rejet du radicalisme analytique et va de pair avec la reconnaissance du hasard ${ }^{20}$.

Autres spécificités objectives par lesquelles l'ordre du vivant se distingue de l'ordre de la matière:

1. La vie est un phénomène singulier, historique et régional. Le temps irréversible de la biologie s'oppose au temps réversible de la physique. La singularité des faits biologiques va de pair avec leur multiplicité et avec leur variabilité ${ }^{21}$. Leur historicité les empêche d'obéir aux lois du déterminisme strict.

2. A la différence des sciences physico-chimiques, les sciences de la vie sont confrontées au problème du Mal. «Il y a là un marqueur rudimentaire, mais robuste, de la différence des ordres pour nous, sinon en soi. L'ordre vivant est normatif, selon Canguilhem ${ }^{22} \gg$ (Fagot-Largeault 2002: 528).

3. L'ordre du vivant ne se laisse pas penser en mathématiques. La biologie se sert sans doute des mathématiques comme instrument (pour la mesure et pour la modélisation expérimentale, par l'intermédiaire de la statistique descriptive pour les enquêtes épidémiologiques), mais les mathématiques ne sont pas son langage. L'outil mathématique est indispensable à la biologie expérimentale pour construire, voire pour concevoir ses modèles, mais la reconstitution des

${ }^{20}$ «On a souvent eu recours à ce concept pour expliquer des phénomènes aussi complexes que la vie, le psychisme et la conscience. En réalité, l'émergence est aussi une caractéristique des systèmes inorganiques. Déjà en 1868, T. H. Huxley soutenait que les propriétés particulières de l'eau, qui lui donnent son caractère 'aqueux' ne pouvaient être déduites des propriétés que l'on connaissait à l'hydrogène et à l'oxygène. Cependant, celui qui utilisa le plus la notion d'émergence fut Llyod Morgan. Il ne fait aucun doute, disait-il, 'qu'à divers degrés d'organisation, les configurations matérielles dévoilent des phénomènes nouveaux et inattendus, et que cela concerne les caractères les plus frappants de la machinerie adaptative'. L'émergence entendue ainsi est quelque chose d'universellement répandu, et, comme l'a dit Popper (Unended Quest), 'nous vivons dans un univers de nouveauté émergente'». Mais «l'émergence est une notion descriptive qui, en particulier dans le cas des systèmes les plus complexes, semble résister à l'analyse. Dire simplement, comme cela a été fait, que l'émergence est la conséquence de la complexité, n'explique rien. Peut-être que les deux plus importantes caractéristiques des 'touts' sont: 1) qu'ils peuvent, à leur tour, devenir des parties de systèmes de niveaux encore plus élevés, et 2) qu'ils peuvent influencer les propriétés des composants aux niveaux inférieurs. Cette dernière est quelquefois appelée 'causalité vers le bas' (B. Campbell (ed.), Human Evolution: An Introduction to Man's Adaptations, Chicago, Aldine, 1974)». E. Mayr, Histoire de la biologie, op. cit., p. 73.

Sur l'histoire du concept d'émergence, cf. M. Mandelbaum, History, Man, and Reason, Baltimore, Johns Hopkins University Press, 1971.

${ }^{21}$ «Tandis que les unités des sciences physiques, disons les atomes ou les particules élémentaires, ont des caractéristiques invariables, les entités biologiques sont déterminées par leur variabilité. Les cellules, par exemple, changent continuellement de propriétés, et cela est vrai aussi des individus. Chaque individu subit un changement drastique, de sa naissance à sa mort, c'est-à-dire depuis le zygote initial, jusqu'à l'adolescence, le stade adulte, la sénescence et la fin. De nouveau, on peut remarquer qu'il n'existe rien de comparable dans le monde inanimé, à l'exception de la décroissance de la radioactivité, du comportement de systèmes complexes (tels le Gulf Stream ou les systèmes climatiques), et de quelques vagues analogies en astrophysique». E. Mayr, Histoire de la biologie, op . cit., p. 65.

${ }^{22}$ A. Fagot-Largeault, in D. Andler, A. Fagot-Largeault, B. Saint-Sernin, Philosophie des sciences, op. cit. , p. 528. 
enchaînements et des rencontres dont dépendent l'imputation causale et l'explication des processus biologiques prend nécessairement la forme d'un récit; elle interdit de penser directement en équations et d'abandonner le langage dit naturel (en fait une variante spécialisée de la langue écrite savante) au profit exclusif de la formalisation mathématique (Husserl 1989: 54) ${ }^{23}$.

Les propriétés par lesquelles l'ordre du vivant se distingue et se sépare de l'ordre physico-chimique se retrouvent dans l'ordre anthropologique. Comme la biologie, la sociologie a affaire à des ensembles, à des «totalités », qui ne se laissent pas réduire aux éléments unitaires qui les composent; tels les organes, les institutions s'acquittent de fonctions (qu'il faut, comme en biologie, distinguer des fins). Comme la biologie, elle oscille entre le holisme et le réductionnisme, en l'espèce l'individualisme méthodologique. Pas plus que le biologiste, le sociologue (non plus, sans doute, que l'ethnologue et que l'historien) ne peut éviter le problème du Mal, pour lui, on l'a vu, beaucoup plus embarrassant. Comme les sciences du vivant, plus encore qu'elles sans doute, les sciences de l'homme sont confrontées à la contingence historique, à la variabilité individuelle, au caractère non-universel, à la faible répétabilité, voire à la singularité des faits qu'elles étudient. Les sciences de l'homme, et pas seulement l'histoire, ne sont évidemment pas moins historiques que la biologie. Si elles se servent, comme la biologie, des mathématiques, elles ne pensent pas davantage qu'elle en équations, mais en concepts. Le passage à la formalisation mathématique se paie, pour elles aussi, d'un écart fatal avec une réalité où le temps est irréversible (Grignon 2001) ${ }^{24}$. Les sciences de l'homme ne peuvent donc s'accommoder de l'épistémologie laplacienne et de la conception mécaniste de la causalité. Mais la reconnaissance de l'existence du hasard n'oblige nullement à renoncer au déterminisme et au principe de causalité, comme y invite le relativisme absolu (qui persiste à considérer le déterminisme strict comme le principe même de l'explication et de la connaissance scientifiques, comme en témoigne en particulier l'interprétation lettrée des révolutions de la physique). «Le hasard détermine», comme dit E. Mayr ${ }^{25}$. Si l'on admet la pluralité, la multiplicité des causes qui concourent à produire un effet $^{26}$,

23 «A l'égard de la forme spatio-temporelle de la nature nous possédons justement le pouvoir 'inné' (comme on l'appellera plus tard) de connaître de façon déterminée l'être en-soi vrai en tant qu'être dans l'idéalité mathématique (avant toute expérience réelle). Ce qui veut dire que l'idéalité mathématique est implicitement innée pour nous. Il en va tout autrement de la légalité concrète universelle de la nature, bien qu'elle soit elle aussi mathématique de fond en comble. Elle est a posteriori, c'est-à-dire accessible inductivement, à partir de la factualité des données d'expérience (von den faktischen Erfahrungsgegebenheiten). Ainsi se trouvent opposées l'une à l'autre, séparées par une coupure nette qui est supposée parfaitement compréhensible, la mathématique apriorique des formes spatiotemporelles et la science de la nature, qui, bien qu'elle applique la mathématique, est inductive. Ou encore: il se fait une séparation tranchée entre la relation purement mathématique de raison à conséquence, et celle de la raison réelle aux conséquences réelles, c'est-à-dire celle de la causalité naturelle». E. Husserl, La crise des sciences européennes et la phénoménologie transcendantale, op. cit., p. 63.

${ }^{24}$ C. Grignon, «La formalisation et les sciences du récit: le cas de la sociologie» in J.-Y. Grenier, C. Grignon, P.-M. Menger (dir.) Le modèle et le récit, op. cit., p. 7-43.

${ }^{25}$ E. Mayr, Histoire de la biologie, op. cit., p. 68.

26 «Déjà Fr. Bacon avait montré que la science ne pouvait s'accommoder que de la vera causa, c'est-à-dire de la cause empirique: la cause d'un phénomène est un autre phénomène, ou plutôt un 
on est amené à reconstituer à la fois «l'ordre des causes » (les enchaînements, les processus) et les «faits de hasard», les occasions, les collisions, les chocs, la succession des rencontres improbables entre des séries causales indépendantes qui aboutissent à la formation d'une conjoncture historique particulière (la révolution française ou aussi bien l'apparition des procaryotes). L'enquête causale procède, on l'a vu, à la reconstitution de l'irréel, elle montre comment, dans quelle mesure ce qui s'est réalisé aurait pu ne pas se produire et comment, dans quelle mesure ce qui ne s'est pas réalisé aurait pu se produire. Reconnaître l'existence du hasard ne borne pas la connaissance scientifique, mais lui ouvre au contraire des perspectives nouvelles d'exploration et de réflexion.

Sous tous ces rapports, la division principale se situe donc entre les sciences du monde physique et les sciences du vivant, plutôt qu'entre les sciences de la nature et les sciences de l'homme. Que les sciences de l'homme se retrouvent du côté et aux côtés de la biologie devrait réconforter les sociologues: nul ne songe à rejeter la biologie en dehors de la science. Mais si l'ordre anthropologique présente les mêmes propriétés objectives que l'ordre du vivant, en quoi les sciences de l'homme et les sciences biologiques se distinguent-elles les unes des autres? N'appartiennent-elles pas à la même espèce épistémologique? En principe, il n'y a là rien qui puisse inquiéter les anthropologues (au sens large du terme). En effet, ce sont les sciences de l'homme qui présentent au plus haut degré les propriétés distinctives par lesquelles l'ensemble sciences de l'homme / sciences de la vie s'écarte des sciences physico-chimiques: irréductibilité du tout aux parties en sociologie, singularité, caractère aléatoire et imprévisible des événements en histoire, diversité et variabilité des cultures en ethnologie, etc. C'est donc du côté anthropologique que devrait se situer le type le plus achevé de l'espèce, les sciences biologiques étant, par une sorte de réductionnisme inversé, appelées à se modeler sur les sciences de l'homme, et, à terme, à les rejoindre. Il est inutile d'insister sur l'irréalisme de ce scénario. En fait, ce sont les sciences biologiques, de beaucoup les plus avancées et les plus confirmées (ne serait-ce que par le succès de leurs applications techniques), qui servent de référence et de modèle, de sorte que l'on a des chances de retrouver, pour l'ensemble sciences de l'homme / sciences de la vie, le réflexe réductionniste qui veut que l'ordre de base explique l'ordre supérieur parce qu'il le conditionne. De ce point de vue, la vie sociale ne serait qu'une forme particulière de vie, et la sociologie devient une subdivision, une annexe de la biologie. Les progrès de la neurobiologie peuvent ainsi renforcer l'idée selon laquelle les propriétés spécifiques des sociétés humaines se réduisent aux propriétés biologiques des individus qui les composent. Le relativisme part du pôle opposé mais arrive au même résultat lorsqu'il étend à l'ensemble du monde zoologique les notions de culture, de société, de langage, d'éducation, retrouvant ainsi, à partir de l'ethnologie, la tradition et les tentations réductionnistes de l'éthologie animale.

Il faut donc se demander s'il existe entre l'ordre du vivant et l'ordre anthropologique des différences objectives qui appellent et justifient une nouvelle division,

concours de phénomènes tel que, s'il y manque une seule circonstance, l'effet ne peut pas se produire, tandis qu'il se produit nécessairement dès qu'elles sont toutes réunies ». E. Goblot, Le système des sciences, le vrai, l'intelligible et le réel, op. cit., p. 219. 
à l'intérieur de la communauté épistémologique qui les oppose à l'ordre physicochimique. Pour Cournot, c'est la capacité d'action réfléchie, consciente et rationnelle, qui caractérise en propre «le règne de l'homme», par opposition au «règne de la Nature »: «Voyez nos digues, nos canaux, nos ports, nos tunnels et comparez les aux assises rocheuses, aux anses du rivage, aux grottes, aux cataractes, aux méandres des cours d'eau naturels. Là on reconnaîtra la main, ou plutôt la marque du génie de l'homme qui procède sur épure à l'aide de la règle et du compas, qui dresse des devis et des calculs, qui est tenu d'opérer avec méthode, nombre et mesure (...) l'on distingue fort bien ce qui se fait selon l'impulsion de la Nature, ce qu'elle peut revendiquer comme venant d'elle, et ce qui a le caractère des œuvres de l'homme, ce qui se fait méthodiquement, logiquement, rationnellement, quoique pas toujours raisonnablement» (Cournot 1987 [1875]: 107sq.) ${ }^{27}$. La rationalité des stratégies individuelles est au principe de l'économie mathématisée, de son axiomatique et de ses tentatives de théorisation, en particulier de la théorie des jeux ${ }^{28}$. Kroeber distingue également entre la vie organique et le niveau supérieur de la culture (équivalent de la civilisation chez Cournot):

\begin{abstract}
'Superorganic' does not mean nonorganic, or free of organic influence and causation; nor does it mean that culture is an entity independent of organic life in the sense that some theologians might assert that there is a soul which is or can become independent of the living body. 'Superorganic' means simply that when we consider culture we are dealing with something that is organic but which must also be viewed as something more than organic if it is to be fully intelligible to us. In the same way when we say that plants and animals are 'organic' we do not thereby try to place them outside the laws of matter and energy in general. We only affirm that fully to understand organic beings and how they behave, we have to recognize certain kinds of phenomena or properties - such as the powers of reproduction, assimilation, irritability - as added to those which we encounter in inorganic substances. Just so, there are certain properties of culture - such as transmissibility, high variability, cumulativeness, value standards, influence on individuals which it is difficult to explain, or to see much significance in, strictly in terms of the organic composition of personalities or individuals. These properties or qualities of culture evidently attach not to the organic individual man as such, but to the actions and the behavior products of societies of men - that is, to culture (Kroeber 1963: 61 sq.) ${ }^{29}$.
\end{abstract}

27 A. A. Cournot, Matérialisme, vitalisme, rationalisme. Etude sur l'emploi des données de la science en philosophie, Paris, Vrin, 1987 [1875], pp. 107 sq.

${ }^{28}$ Ou plutôt des théories des jeux : «La théorie des jeux est une représentation mathématique axiomatique, formelle, abstraite des comportements subjectifs qui a pour but d'essayer de représenter la logique du comportement des sujets humains, d'en faire des simulations, au lieu de se proposer l'objectif démesuré de donner une description analytique des causes de ce comportement. C'est essentiellement là le point de vue de von Neumann (...). Mais l'histoire de la théorie des jeux ne se réduit pas à l'intervention de von Neumann (...) elle montre qu'il y a eu au moins trois théories des jeux différentes (...) Les théories de Emile Borel, de von Neumann et Morgenstern, et de Nash sont effectivement en conflit entre elles; non seulement en conflit pour ce qui concerne le point de vue initial mais même pour ce qui concerne les résultats mathématiques». G. Israel, «La représentation formelle des comportements subjectifs: le cas de la théorie des jeux », in C. Grignon, C. Kordon, ed., L'ordre des sciences, essais d'épistémologie comparée, Paris, Editions de la Maison des Sciences de l'Homme, à paraître.

29 A. L. Kroeber, Anthropology, Culture Patterns and Processes, New-York, Harcourt Brace Jovanovitch, 1963, p. 61 sq. (première formulation, 1917). 
Par ordres de réalité, on entend ici les niveaux d'organisation, plus ou moins élevés, auquel le savant choisit, comme on l'a vu, de s'intéresser à l'intérieur de chacun des domaines de réalité concrets; par exemple, s'agissant des êtres vivants, aux molécules, ou aux organismes, s'agissant de l'homme, à la chimie du cerveau ou aux institutions. C'est son point de vue qui fait l'objet, i.e. qui détermine les problèmes à résoudre, la manière de travailler et de raisonner, et les divisions même des sciences en disciplines. C'est pourquoi l'opposition entre le «monde inanimé » et le «monde du vivant» ne débouche pas directement sur une typologie des sciences et ne recouvre pas exactement l'opposition entre d'une part les sciences nomothétiques et expérimentales, et, de l'autre, les sciences historiques et observationnelles. Comme on l'a vu, la biologie est partagée entre la micro-biologie et les macro-biologies, biologie des organismes, biologie des populations, biologie de l'évolution. Les sciences les plus capables de monter des expériences recourent par là-même à l'observation; l'astronomie repose sur l'observation expérimentale. Comme le notait déjà Whewell, les problèmes de classification, centraux dans les sciences de l'homme, en particulier en ethnologie et en archéologie, se retrouvent en minéralogie et en botanique. Les équations de la physique contemporaine demeurent réversibles; mais la cosmologie, comme l'archéologie ou la paléontologie, forme et vérifie ses hypothèses à partir de vestiges («rayonnement fossile»). Du reste les sciences nomothétiques sont toujours pour une part, à leur manière, des sciences historiques. «Dans chaque loi il faut distinguer la relation mathématique, et les constantes qui sont des données de fait (par exemple dans la loi de Newton). Chaque science théorique, sauf les mathématiques, se double d'une science historique. Quand on peut remonter de l'état final ou de l'état pénultième d'un système à l'état antérieur et de proche en proche à l'état initial, l'histoire n'intervient pas; le système s'est développé pour ainsi dire en dehors du temps. Mais le plus souvent, pour expliquer l'état actuel d'un système (par exemple le système astronomique) il faut faire appel à des faits indépendants de la théorie, qui ne s'expliquent pas par elle, qu'on ne pouvait prévoir, etc. Exemple: la position initiale des astres est une donnée historique, irréductible aux lois mécaniques de l'astronomie» (Lalande 1960 [1926]) ${ }^{30}$.

Mais une fois qu'on a choisi le niveau d'organisation auquel on s'intéresse, c'est l'objet qui détermine le point de vue. Les différences entre les ordres de la réalité, ainsi définis, requièrent des manières différentes de faire et donc de concevoir la science. Plus la diversité des phénomènes étudiés est grande, et plus les tâches d'inventaire, de collecte, de description et de classement sont prioritaires. Dans l'ordre du vivant, plus encore dans l'ordre anthropologique, la prépondérance des «données historiques» par rapport aux «données théoriques» (Cournot) oblige les sciences à extraire la connaissance des faits par induction («le processus par lequel on récolte des vérités générales à partir de l'examen des faits particuliers ») (Whewell 1837) ${ }^{31}$; elle empêche de partir d'une axiomatique, retarde le passage à la méthode déductive, interdit le recours exclusif à la formalisation logico-mathématique. En biologie comme dans les sciences de l'homme,

${ }^{30}$ A. Lalande, op. cit., article «Histoire», observations de F. Mentré.

${ }^{31} \mathrm{~W}$. Whewell, History of the Inductive Sciences, from the earliest to the present Time, London, Parker, 1837. 
la diversité et la multiplicité des phénomènes obligent à abstraire, à conceptualiser et à généraliser progressivement. La limitation de la capacité prédictive conduit à reconnaître l'existence du hasard. L'irréversibilité du temps contraint, pour expliquer, à reconstituer des processus ; l'imputation causale prend nécessairement la forme d'un récit. Par rapport à l'épistémologie idéale des sciences physiques, le degré supérieur d'organisation des ordres du vivant semble ainsi limiter les possibilités et les ambitions des sciences biologiques et des sciences de l'homme; mais les difficultés supplémentaires que celles-ci rencontrent leur permettent d'imaginer et de développer des méthodes et des techniques nouvelles, de poser d'autres problèmes, de reformuler des questions centrales, comme celle du déterminisme et de la causalité. «Le monde des êtres vivants est bien plus riche que celui des objets inanimés, et une philosophie de la science dérivée des sciences physiques ignore presque entièrement de vastes régions de la structure conceptuelle de la biologie» (Mayr 1981: 91) ${ }^{32}$. La référence à l'idéal des sciences nomothétiques enferme les sciences de l'homme dans la spéculation en encourageant, sous couvert d'impeccabilité scientifique, le goût pour les généralités et la «philosophie paresseuse », au détriment de la recherche des faits; pour qu'elles cessent de confondre théories et systèmes de pensée, il faut au contraire qu'elles reconnaissent leur spécificité épistémologique et admettent qu'elles sont pour ainsi dire le type idéal des sciences historiques et narratives, fondées sur l'observation, l'induction et la conceptualisation progressive.

\footnotetext{
${ }^{32}$ E. Mayr, La biologie de l'évolution, Paris, Hermann, 1981, p. 91.
} 